\title{
Targeting splicing factors as molecular non-muscle invasive bladder cancer predictors
}

\author{
Kyoung-Hwa Lee, Ja Hyeon Ku \\ Department of Urology, Seoul National University Hospital, Seoul, Republic of Korea \\ Correspondence to: Ja Hyeon Ku, MD, PhD. Department of Urology, Seoul National University Hospital, 101 Daehak-ro, Jongno-gu, Seoul 110-744, \\ Republic of Korea. Email: kuuro70@snu.ac.kr. \\ Provenance: This is an Invited Editorial commissioned by Section Editor Xiao Li (Department of Urology, Jiangsu Cancer Hospital \& Jiangsu \\ Institute of Cancer Research \& Nanjing Medical University Affiliated Cancer Hospital, Nanjing, China). \\ Comment on: Bielli P, Panzeri V, Lattanzio R, et al. The Splicing Factor PTBP1 Promotes Expression of Oncogenic Splice Variants and Predicts Poor \\ Prognosis in Patients with Non-muscle-Invasive Bladder Cancer. Clin Cancer Res 2018;24:5422-32.
}

Submitted Oct 05, 2018. Accepted for publication Oct 12, 2018.

doi: $10.21037 /$ tau.2018.10.09

View this article at: http://dx.doi.org/10.21037/tau.2018.10.09

Bladder cancer is the fourth most common cancer in men, with an estimated diagnosis of 60,490 new cases and 12,240 deaths in the United States in 2017 (1). Nearly $75 \%$ of bladder cancer patients are diagnosed with nonmuscle invasive bladder cancer (NMIBC), which consists of stages Ta, T1, and carcinoma in situ (CIS). NMIBC is rarely lethal, but $50-70 \%$ of patients experience disease recurrence and $10-30 \%$ of patients' progress to lifethreatening muscle-invasive bladder cancer (MIBC) (2). Therefore, early diagnosis of NMIBC and implementation of proper treatment are essential for positive outcomes. A combination of cystoscopy, voided urinary cytology, and imaging is the current standard diagnosis for NMIBC, but these tools have certain limitations. To address these limitations, numerous candidate protein markers have been tested and approved by the U.S Food and Drug Administration (FDA) (3). Based on the molecular markers and protein expression, NMIBC is segregated into two subtypes as low-risk subtype class 1 (UroA, -CIS signature, -progression signature) and high-risk subtype class 2 (GU/SCCL, +CIS signature, +progression signature). However, Hedegaard et al. demonstrated an absence of correlation between these subtypes and treatment response (4). Consequently, new tools for identifying NMIBC stages, determining the probability of recurrence, and ascertaining drug sensitivity would be beneficial for the development of targeted drugs and the improvement in patient outcomes.

Among many biological processes affecting tumor progression, alternative splicing was identified as a key process in many cancers through massive systematic genomic analysis (5). Splicing is the removal of introns from pre-mRNA during transcription, resulting in mature mRNA. This process is catalyzed by the spliceosome, a large complex composed of five small nuclear ribonucleoproteins (snRNPs: U1, U2, U4, U5, and U6) and several associated protein cofactors. Alternative splicing can generate a variety of protein isoforms from a single gene by including or excluding whole or partial exons, resulting in different versions of the mature mRNA. Approximately, three-quarters of human genes undergo alternative splicing. Recently, several studies have established alternative splicing as a cancer initiation and maintenance factor $(5,6)$. The splicing pattern of specific isoforms of cancerrelated genes undergoes alteration as cells go through the oncogenic process of gaining proliferation capacity and acquiring angiogenic, invasive, antiapoptotic, and survival properties. Furthermore, RNA-sequencing analysis of tumor tissues compared with normal tissues from the same individuals has revealed more global features of alternative splicing. Previous studies have demonstrated the oncogenic functions of splicing pattern and splicing-related proteins (7-9) and the proteins hold the potential to be considered as promising targets for anticancer drug development.

Among the heterogeneous nuclear ribonucleoproteins (hnRNPs) comprising the spliceosome, the oncogenic function of PTBP1 (also known as hnRNPI) in bladder 
cancer was recently determined. Pamela et al. reported expression changes in PTBP1 in NMIBC (10). They identified PTBP1 from a public database as a candidate factor linked with progression of bladder cancer. As per the datasets on bladder cancer, increase in the PTBP1 mRNA expression has been reported compared to normal tissue, and Kaplan-Meier analysis revealed a significant association between high PTBP1 mRNA expression and low overall survival (OS) probability. The mRNA expression of other splicing factors (SFs) remained unchanged in the same datasets. To confirm this result at the protein level, the researchers screened 152 bladder cancer tissues using PTBP1 antibody immuno-staining technique and 50 bladder cancer tissues using the western blotting technique. Both the methods showed that higher PTBP1 protein expression was correlated with higher tumor stages and a higher risk of cancer progression. The survival ratio of PTBP1-high cancer patients was lower than that of PTBP1low cancer patients. Knockdown of PTBP1 gene expression in bladder cancer cell lines resulted in enhancement of growth retardation and cell death in all the tested bladder cancer cells. Interestingly, silencing of PTBP1 led to an increase in drug sensitivity of bladder cancer cells.

Bielli et al. (10) examined splicing variations in eight prooncogenic genes within PTBP1 knockdown bladder cancer cells. The eight genes studied were PKM, NUMB (cell proliferation), FAS (cell death), ACTN1, MACF1, TPM1, CTNND1, and CD44 (cell migration and adhesion). PTBP1 knockdown altered splicing of the pro-oncogenic variant of all the tested bladder cancer-related target genes. The authors revealed that this process was accompanied by direct binding of PTBP1 on pre-mRNAs in vivo based on a UV-crosslink and RNA immunoprecipitation (CLIP) assay. Finally, the authors analyzed 18 NMIBC patients' tissues for PTBP1 expression and CD44 variable exon splicing patterns. High expression of PTBP1 was correlated with the inclusion of $\mathrm{v} 5$ and $\mathrm{v} 7$ exons of CD44. This result indicated the influence of PTBP1 level on splicing outcome.

Numerous studies have been attempted to find inhibitors that target the spliceosome and that can be used as anticancer drugs in diverse type of cancers. Some chemical compounds, including spliceostatin A, herboxidenes, and pladienolides target the SF3B component of U2 snRNP $(11,12)$, and have demonstrated potent cytotoxicity and ability to arrest cell cycle arrest. The compounds have been reported to interrupt early stages of spliceosome assembly, resulting in accumulation of pre-mRNA into the cytoplasm thus demonstrating the possibility of targeting other spliceosome-associated enzymes or alternative splicing in the development of anti-cancer drugs. Studies on targeting SF3B with the E7107 compound were attempted in Europe (Study E7107-A001-101) and the U.S. (Trial registration ID: NCT00499499). The results of the reported studies have been encouraging, but the unexpected toxicity of bilateral optic neuritis was identified and resulted in the suspension of both the trials $(13,14)$. Although it is not clear whether this toxicity is an on-target effect of SF3B1 inhibition or a specific toxicity associated with E7107, it is hypothesized that targeting more specific spliceosome enzymes rather than SF3B1 would increase drug specificity and reduce toxicity in cancer patients. According to Pamela et al.'s study, another possible target is PTBP1. This approach is especially promising for NMIBC as the authors demonstrated NMIBC-specific expression changes in the PTBP1 enzyme and the reduction in NMIBC proliferation by targeting PTBP1.

Despite the presence of remarkable findings, the literature lacks complete results on patient outcome after cancer drug treatment targeting PTBP1 expression. The possible reason could be the limited sample size of patient tissues. It is hypothesized that large-scale studies in the future dealing with comparison of PTBP1 expression levels and anti-cancer drug response would be valuable for screening suitable NMIBC drug treatment.

\section{Acknowledgements}

Funding: This study was supported by the Basic Science Research Program through the National Research Foundation of Korea (NRF) funded by the Ministry of Education (No. 2016R1D1A1A02936950) and the Korea government (MSIP) (No. 2016R1A2B4011623).

\section{Footnote}

Conflicts of Interest: The authors have no conflicts of interest to declare.

\section{References}

1. Siegel RL, Miller KD, Jemal A. Cancer Statistics, 2017. CA Cancer J Clin 2017;67:7-30.

2. Kamat AM, Hahn NM, Efstathiou JA, et al. Bladder cancer. Lancet 2016;388:2796-810.

3. Bryan RT, Zeegers MP, James ND, et al. Biomarkers in bladder cancer. BJU Int 2010;105:608-13. 
4. Hedegaard J, Lamy P, Nordentoft I, et al. Comprehensive Transcriptional Analysis of Early-Stage Urothelial Carcinoma. Cancer Cell 2016;30:27-42.

5. Lee SC, Abdel-Wahab O. Therapeutic targeting of splicing in cancer. Nat Med 2016;22:976-86.

6. Oltean S, Bates DO. Hallmarks of alternative splicing in cancer. Oncogene 2014;33:5311-8.

7. Karni R, de Stanchina E, Lowe SW, et al. The gene encoding the splicing factor SF2/ASF is a proto-oncogene. Nat Struct Mol Biol 2007;14:185-93.

8. Anczukow O, Rosenberg AZ, Akerman M, et al. The splicing factor SRSF1 regulates apoptosis and proliferation to promote mammary epithelial cell transformation. Nat Struct Mol Biol 2012;19:220-8.

9. David CJ, Chen M, Assanah M, et al. HnRNP proteins controlled by c-Myc deregulate pyruvate kinase mRNA splicing in cancer. Nature 2010;463:364-8.

10. Bielli P, Panzeri V, Lattanzio R, et al. The Splicing Factor PTBP1 Promotes Expression of Oncogenic Splice Variants

Cite this article as: Lee $\mathrm{KH}, \mathrm{Ku} \mathrm{JH}$. Targeting splicing factors as molecular non-muscle invasive bladder cancer predictors. Transl Androl Urol 2018;7(Suppl 6):S715-S717. doi: 10.21037/ tau.2018.10.09 and Predicts Poor Prognosis in Patients with Non-muscleInvasive Bladder Cancer. Clin Cancer Res 2018;24:5422-32.

11. Kaida D, Motoyoshi H, Tashiro E, et al. Spliceostatin A targets SF3b and inhibits both splicing and nuclear retention of pre-mRNA. Nat Chem Biol 2007;3:576-83.

12. Kotake Y, Sagane K, Owa T, et al. Splicing factor SF3b as a target of the antitumor natural product pladienolide. Nat Chem Biol 2007;3:570-5.

13. Eskens FA, Ramos FJ, Burger H, et al. Phase I pharmacokinetic and pharmacodynamic study of the firstin-class spliceosome inhibitor E7107 in patients with advanced solid tumors. Clin Cancer Res 2013;19:6296-304.

14. Hong DS, Kurzrock R, Naing A, et al. A phase I, openlabel, single-arm, dose-escalation study of E7107, a precursor messenger ribonucleic acid (pre-mRNA) splicesome inhibitor administered intravenously on days 1 and 8 every 21 days to patients with solid tumors. Invest New Drugs 2014;32:436-44. 